\title{
Anti-AIDS agents 87. New bio-isosteric dicamphanoyl- dihydropyranochromone (DCP) and dicamphanoyl-khellactone (DCK) analogues with potent anti-HIV activity
}

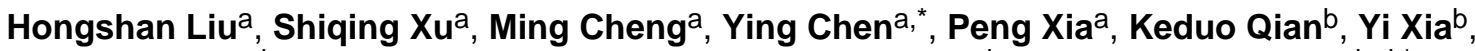 \\ Zheng-Yu Yang ${ }^{b}$, Chin-Ho Chen ${ }^{c}$, Susan L. Morris-Natschke ${ }^{b}$, and Kuo-Hsiung Lee ${ }^{b, d, *}$ \\ aDepartment of Medicinal Chemistry, School of Pharmacy, Fudan University, Shanghai 201203, \\ China \\ bNatural Products Research Laboratories, UNC Eshelman School of Pharmacy, University of \\ North Carolina, Chapel Hill, NC 27599-7568, USA \\ 'Duke University Medical Center, Durham, NC 27710, USA \\ ${ }^{\mathrm{d} C h i n e s e ~ M e d i c i n e ~ R e s e a r c h ~ a n d ~ D e v e l o p m e n t ~ C e n t e r, ~ C h i n a ~ M e d i c a l ~ U n i v e r s i t y ~ a n d ~ H o s p i t a l, ~}$ \\ Taichung, Taiwan
}

\begin{abstract}
Six 3'R,4'R-di- $O$-(S)-camphanoyl-2',2'-dimethyldihydropyrano[2,3-f]chromone (DCP) and two $3^{\prime} R, 4^{\prime} R$-di- $O$ - $(S)$-camphanoyl-(+)-cis-khellactone (DCK) derivatives were designed, synthesized, and evaluated for inhibition of HIV-1 ${ }_{\mathrm{NL} 4-3}$ replication in TZM-bl cells. 2-Ethyl-2'-monomethyl-1'oxa- and -1'-thia-DCP (5a, 6a), as well as 2-ethyl-1'-thia-DCP (7a) exhibited potent anti-HIV activity with $\mathrm{EC}_{50}$ values of 30,38 and $54 \mathrm{nM}$ and therapeutic indexes of 152.6, 48.0 and 100.0, respectively, which were better than or comparable to those of the lead compound 2-ethyl-DCP in the same assay. 4-Methyl-1'-thia-DCK (8a) also showed significant inhibitory activity with an $\mathrm{EC}_{50}$ of $128 \mathrm{nM}$ and TI of 237.9 .
\end{abstract}

\section{Keywords}

2'-Monomethyl-1'-oxa-DCP; 2'-Monomethyl-1'-thia-DCP; 2-Ethyl-1'-thia-DCP; 4-Methyl-1'-thiaDCK; Anti-HIV activity

\begin{abstract}
In our previous research, $3^{\prime} R, 4^{\prime} R$-di- $O$-(S)-camphanoyl-(+)-cis-khellactone (DCK, 1, Fig. 1) demonstrated extremely potent inhibitory activity against HIV-1 replication in H9 lymphocytic cells. ${ }^{1}$ Subsequently, hundreds of DCK and some of its ring-A positional isomer DCP ( $3^{\prime} R, 4^{\prime} R$-di- $O$ - $(S)$-camphanoyl-2',2'-dimethyl-dihydro-pyrano[2,3-f]chromone, 2, Fig. 1) derivatives have been designed, synthesized and screened for anti-HIV activity in H9 lymphocytes, MT-2 cell lines, and MT-4 cell lines. ${ }^{2-8}$ 4-Methyl-DCK (3, Fig.1) and 2ethyl-DCP (4, Fig.1) showed the most promising anti-HIV results in these two series.
\end{abstract}

\footnotetext{
(C) 2011 Elsevier Ltd. All rights reserved.

*Corresponding authors. Tel.: +86 2151980116 (Y.C.); Tel.: +1 919962 0066; Fax: +1 9199663893 (K.H.L.); yingchen71@fudan.edu.cn; khlee@unc.edu.

Publisher's Disclaimer: This is a PDF file of an unedited manuscript that has been accepted for publication. As a service to our customers we are providing this early version of the manuscript. The manuscript will undergo copyediting, typesetting, and review of the resulting proof before it is published in its final citable form. Please note that during the production process errors may be discovered which could affect the content, and all legal disclaimers that apply to the journal pertain.
} 
Structure-activity relationship (SAR) studies found that DCP derivatives exhibited better anti-HIV activity than the corresponding DCKs; ${ }^{8} 2^{\prime}$ - $\alpha$-monomethyl-4-methyl DCK derivatives were more potent than $2^{\prime}$-gem-dimethyl DCKs; ${ }^{9}$ bio-isosteric analogues with a sulfur rather than oxygen in the ring-C of DCK exhibited remarkable inhibitory effects on HIV-1 replication; ${ }^{9,10}$ and a $3^{\prime}, 4^{\prime}$-dicamphanoyl moiety is indispensable for anti-HIV activity. ${ }^{11}$ Considering these SAR research results, we have now designed and synthesized 2'-monomethyl-DCP (5, 1'-oxa; 6, 1'-thia), 2-ethyl-1'-thia-DCP (7), and 4-methyl-1'-thiaDCK (8) analogues to further explore the pharmacophores of the 2 '-position and the bioisosteric effect at the 1'-position. This paper reports their synthesis and anti-HIV bioassay data.

The synthetic routes to $\mathbf{5 a}, \mathbf{5 b}, \mathbf{6 a}$ and $\mathbf{6 b}$ are shown in Scheme 1. The intermediate 2ethyl-7-mercapto- $4 \mathrm{H}$-chromen-4-one (12) was obtained by reacting 2-ethyl-7-hydroxy- $4 \mathrm{H}$ chromen-4-one (9) with dimethylthiocarbamoyl chloride in EtOH in the presence of anhydrous potassium carbonate, followed by a rearrangement at $240{ }^{\circ} \mathrm{C}$, then hydrolysis with methanolic $\mathrm{KOH}$ and acidification with $\mathrm{HCl}$. Compounds 9 and $\mathbf{1 2}$ were treated with 3chloro-1-butyne in dimethyl formamide (DMF) or acetone in the presence of anhydrous potassium carbonate and potassium iodide at room temperature to produce the propargyl ethers $\mathbf{1 3}$ and 14, followed by thermal rearrangement in refluxing $N, N$-diethylaniline to form intermediates $\mathbf{1 5}$ and 16. Sharpless dihydroxylation (AD) of $\mathbf{1 5}$ and $\mathbf{1 6}$ afforded dihydroxy derivatives 17a/17b and 18a/18b, respectively, as diastereoisomeric mixtures. Target compounds $\mathbf{5 a}$ and $\mathbf{5 b}$ were obtained by acylation of $\mathbf{1 7} \mathbf{a}$ and $\mathbf{1 7} \mathbf{b}$ with $(S)$-(-)-camphanic chloride in $\mathrm{CH}_{2} \mathrm{Cl}_{2}$ at room temperature with 4-dimethylaminopyridine (DMAP) as acid scavenger. Compounds $6 \mathbf{a}$ and $6 \mathbf{b}$ were synthesized by the same procedure from 18a and 18b. The pure diastereoisomers $5 \mathbf{a}, \mathbf{5 b}, \mathbf{6 a}$, and $\mathbf{6 b}$ were obtained by separation with column chromatography on silica gel [petroleum ether/ethyl acetate, 3:1 (v/v)].

The preparation of 7a and $\mathbf{7 b}$ is illustrated in Scheme 2. 2-Ethyl-7-mercapto-4H-chromen-4one (12) was treated with 3-chloro-3-methyl-1-butyne in $\mathrm{EtOH} / \mathrm{H}_{2} \mathrm{O}(\mathrm{v} / \mathrm{v}=1: 1)$ in the presence of potassium hydroxide at room temperature to produce the propargyl ether 19, followed by thermal rearrangement in refluxing $N, N$-diethylaniline to form intermediate 20 . Sharpless AD of $\mathbf{2 0}$ afforded dihydroxy derivatives $\mathbf{2 1 a}$ and $\mathbf{2 1 b}$. Target compounds $\mathbf{7 a}$ and $\mathbf{7 b}$ were obtained by acylation of $\mathbf{2 1 a}$ and $\mathbf{2 1 b}$ with $(S)$-(-)-camphanic chloride in $\mathrm{CH}_{2} \mathrm{Cl}_{2}$ at room temperature with DMAP as an acid scavenger. Diastereoisomers $\mathbf{7 a}$ and $\mathbf{7 b}$ could be separated by column chromatography on silica gel [petroleum ether/ethyl acetate, 3:1 (v/v)].

The synthesis of $\mathbf{8 a}$ and $\mathbf{8 b}$ was accomplished by a similar four-step sequence, as depicted in Scheme 3. Diastereoisomers 8a and $\mathbf{8 b}$ were separated by HPLC on an Alltima column (2.1 $\mathrm{mm} \times 150 \mathrm{~mm}, \mathrm{C}-18)$ with acetonitrile/water 70:30 (v/v) as eluant.

The eight newly synthesized compounds $\mathbf{5 - 8} \mathbf{8}^{12}$ were evaluated for anti-HIV activity in TZM-bl cells in parallel with 2-ethyl-DCP. ${ }^{13}$ The bioassay data are summarized in Table 1. Compounds 5a, 6a, and 7a showed significant anti-HIV activity with $\mathrm{EC}_{50}$ values of 30, 38 and $54 \mathrm{nM}$, which were better than the reference compound (2-ethyl-DCP, $\mathrm{EC}_{50}: 120 \mathrm{nM}$ ), and had good therapeutic index (TI) values of 152.6, 48.0 and 100.0, respectively. With a two-fold lower $\mathrm{EC}_{50}$ value, 2-ethyl-1'-thia-DCP (7a) was more potent than 4-methyl-1'-thiaDCK (8a). This result was coincident with the previous activity comparison between the DCP and DCK series, e.g. 2-ethyl DCP was more active than 4-methyl DCK. ${ }^{8} 2^{\prime}$ Monomethyl-2-ethyl-1'-oxa- (5a) and -1'-thia-DCP derivatives (6a) exhibited better antiHIV activity than the corresponding 2'-gem-dimethyl substituted compounds 2-ethyl-DCP and $7 \mathbf{a}$. Interestingly, $\mathbf{5 b}, \mathbf{6 b}, \mathbf{7 b}$ and $\mathbf{8 b}$ exhibited remarkably reduced or even completely abolished anti-HIV activity, consistent with the results from prior compounds. This finding 
suggested that, just as in the DCK series, the spatial orientations of the 2'-methyl group and the $3^{\prime}, 4^{\prime}$-dicamphanoyls are also crucial to anti-HIV activity in DCP analogues.

\section{Supplementary Material}

Refer to Web version on PubMed Central for supplementary material.

\section{Acknowledgments}

This research was supported by grants from the National Natural Science Foundation of China awarded to Y. Chen (No. 30200348 and 30873164) and P. Xia (No. 20272010), respectively, and Grant AI-33066 from the National Institute of Allergies and Infectious Diseases awarded to K.H.L. Thanks are also due to the National Drug Innovative Program 2009ZX09301-011) for partial support.

\section{References}

1. Huang L, Kashiwada Y, Cosentino LM, Fan S, Chen CH, McPhail AT, Fujioka T, Mihashi K, Lee KH. J Med Chem. 1994; 37:3947. [PubMed: 7525962]

2. Takeuchi Y, Xie L, Cosentino LM, Lee KH. Bioorg Med Chem Lett. 1997; 7:2573.

3. Xie L, Takeuchi Y, Cosentino LM, Lee KH. Bioorg Med Chem Lett. 1998; 8:2151. [PubMed: 9873504]

4. Xie L, Takeuchi Y, Concentino LM, McPhail AT, Lee KH. J Med Chem. 2001; 44:664. [PubMed: 11262077]

5. Zhang Q, Chen Y, Xia P, Xia Y, Yang ZY, Yu DL, Morris-Natschke SL, Lee KH. Bioorg Med Chem Lett. 2004; 14:5855. [PubMed: 15501055]

6. Wang Y, Huang SX, Xia P, et al. Bioorg Med Chem Lett. 2007; 17:4316. [PubMed: 17533128]

7. Yu DL, Brossi A, Kilgore N, Wild C, Allaway G, Lee KH. Bioorg Med Chem Lett. 2003; 13:1575. [PubMed: 12699758]

8. Yu DL, Chen CH, Brossi A, Lee KH. J Med Chem. 2004; 47:4072. [PubMed: 15267246]

9. Xu SQ, Yan X, Chen Y, Xia P, Qian KD, Yu DL, Xia Y, Yang ZY, Morris-Natschke SL, Lee KH. Bioorg Med Chem. 2010; 18:7203. [PubMed: 20846868]

10. Chen Y, Zhang Q, Zhang BN, Xia P, Xia Y, Yang ZY, Kilgore N, Wild C, Morris-Natschke SL, Lee KH. Bioorg Med Chem. 2004; 12:6383. [PubMed: 15556756]

11. Xie L, Takeuchi Y, Cosentino LM, Lee KH. J Med Chem. 1999; 42:2662. [PubMed: 10411486]

12. Analytical data of target compounds 5-8: Configuration assignments of isomeric compound pairs were based on prior data in reference 9.5a. $\mathrm{mp} 138-141^{\circ} \mathrm{C} ;{ }^{1} \mathrm{H} \mathrm{NMR}\left(\mathrm{CDCl}_{3}, 400 \mathrm{MHz}\right) \delta 0.95-$ $1.12\left(18 \mathrm{H}, \mathrm{m}, 6 \times-\mathrm{CH}_{3}\right.$ in camphanoyl), 1.67-2.50 (8H, m, 4×- $\mathrm{CH}_{2}$ in camphanoyl), 1.24 (3H, t, $\mathrm{CH}_{3}$ in ethyl), $1.48\left(3 \mathrm{H}, \mathrm{d}, J=6.3 \mathrm{~Hz}, 2^{\prime}-\mathrm{CH}_{3}\right), 2.56\left(2 \mathrm{H}, \mathrm{m},-\mathrm{CH}_{2}\right.$ in ethyl $), 4.55\left(1 \mathrm{H}, \mathrm{m}, 2^{\prime}-\mathrm{CH}\right)$, $5.17\left(1 \mathrm{H}, \mathrm{m}, 3^{\prime}-\mathrm{H}\right), 6.14(1 \mathrm{H}, \mathrm{s}, 3-\mathrm{H}), 6.80\left(1 \mathrm{H}, \mathrm{d}, J=3.1 \mathrm{~Hz}, 4^{\prime}-\mathrm{H}\right), 6.93(1 \mathrm{H}, \mathrm{d}, J=9.0 \mathrm{~Hz}, 6-\mathrm{H})$, $8.12(1 \mathrm{H}, \mathrm{d}, J=9.0 \mathrm{~Hz}, 5-\mathrm{H}) .[\alpha]_{\mathrm{D}}-37\left(c 0.1, \mathrm{CHCl}_{3}\right)$. HRMS(MALDI-DHB): Calcd. for $\mathrm{C}_{35} \mathrm{H}_{40} \mathrm{O}_{11}$ : 636.2571; Found: $637.2643\left[\mathrm{M}+\mathrm{H}^{+}\right]$. 5b. mp $203-206^{\circ} \mathrm{C} ;{ }^{1} \mathrm{H} \mathrm{NMR}\left(\mathrm{CDCl}_{3}, 400\right.$ $\mathrm{MHz}) \delta 0.85\left(3 \mathrm{H}, \mathrm{s},-\mathrm{CH}_{3}\right.$ in camphanoyl), 1.02-1.13 (15H, m, $-\mathrm{CH}_{3} \times 5$ in camphanoyl), 1.68 $2.41\left(8 \mathrm{H}, \mathrm{m}, 4 \times-\mathrm{CH}_{2}\right.$ in camphanoyl), $1.29\left(3 \mathrm{H}, \mathrm{t},-\mathrm{CH}_{3}\right.$ in ethyl $), 1.47\left(3 \mathrm{H}, \mathrm{d}, J=6.3 \mathrm{~Hz}, 2^{\prime}-\right.$ $\left.\mathrm{CH}_{3}\right), 2.47\left(2 \mathrm{H}, \mathrm{m},-\mathrm{CH}_{2}\right.$ in ethyl), $4.66\left(1 \mathrm{H}, \mathrm{m}, 2^{\prime}-\mathrm{CH}\right), 5.29\left(1 \mathrm{H}, \mathrm{m}, 3^{\prime}-\mathrm{H}\right), 6.14(1 \mathrm{H}, \mathrm{s}, 3-\mathrm{H})$, $6.81\left(1 \mathrm{H}, \mathrm{d}, J=3.5 \mathrm{~Hz}, 4^{\prime}-\mathrm{H}\right), 6.92(1 \mathrm{H}, \mathrm{d}, J=8.6 \mathrm{~Hz}, 6-\mathrm{H}), 8.11(1 \mathrm{H}, \mathrm{d}, J=9.0 \mathrm{~Hz}, 5-\mathrm{H}) .[\alpha]_{\mathrm{D}}$ +81 ( $c$ 0.1, $\mathrm{CHCl}_{3}$ ). HRMS(MALDI-DHB): Calcd. for $\mathrm{C}_{35} \mathrm{H}_{40} \mathrm{O}_{11}: 636.2571$; Found: 637.2643 $\left[\mathrm{M}+\mathrm{H}^{+}\right]$. 6a. $\mathrm{mp} 175-178^{\circ} \mathrm{C} ;{ }^{1} \mathrm{H} \mathrm{NMR}\left(\mathrm{CDCl}_{3}, 400 \mathrm{MHz}\right) \delta 0.98-1.13\left(18 \mathrm{H}, \mathrm{m}, 6 \times-\mathrm{CH}_{3}\right.$ in camphanoyl), $1.68-2.59\left(8 \mathrm{H}, \mathrm{m}, 4 \times-\mathrm{CH}_{2}\right.$ in camphanoyl), $1.25\left(3 \mathrm{H}, \mathrm{t},-\mathrm{CH}_{3}\right.$ in ethyl), $1.37(3 \mathrm{H}, \mathrm{d}$, $\left.J=6.7 \mathrm{~Hz}, 2^{\prime}-\mathrm{CH}_{3}\right), 2.47\left(2 \mathrm{H}, \mathrm{m},-\mathrm{CH}_{2}\right.$ in ethyl), $3.87\left(1 \mathrm{H}, \mathrm{m}, 2^{\prime}-\mathrm{CH}\right), 5.32\left(1 \mathrm{H}, \mathrm{m}, 3^{\prime}-\mathrm{H}\right), 6.15$ $(1 \mathrm{H}, \mathrm{s}, 3-\mathrm{H}), 6.97\left(1 \mathrm{H}, \mathrm{d}, J=2.7 \mathrm{~Hz}, 4^{\prime}-\mathrm{H}\right), 7.12(1 \mathrm{H}, \mathrm{d}, J=8.6 \mathrm{~Hz}, 6-\mathrm{H}), 8.05(1 \mathrm{H}, \mathrm{d}, J=8.6 \mathrm{~Hz}$, 5-H). $[\alpha]_{D}-272$ ( $c 0.1, \mathrm{CHCl}_{3}$ ). HRMS(MALDI-DHB): Calcd. for $\mathrm{C}_{35} \mathrm{H}_{40} \mathrm{O}_{10} \mathrm{~S}: 675.2240[\mathrm{M}$ $+\mathrm{Na}^{+}$; Found: $675.2234\left[\mathrm{M}+\mathrm{Na}^{+}\right]$. 6b. $\mathrm{mp} 152-155^{\circ} \mathrm{C} ;{ }^{1} \mathrm{H} \mathrm{NMR}\left(\mathrm{CDCl}_{3}, 400 \mathrm{MHz}\right) 0.82-1.13$ $\left(18 \mathrm{H}, \mathrm{m},-\mathrm{CH}_{3} \times 6\right.$ in camphanoyl $), 1.68-2.66\left(8 \mathrm{H}, \mathrm{m}, 4 \times-\mathrm{CH}_{2}\right.$ in camphanoyl $), 1.29\left(3 \mathrm{H}, \mathrm{t},-\mathrm{CH}_{3}\right.$ in ethyl), $1.38\left(3 \mathrm{H}, \mathrm{d}, J=6.7 \mathrm{~Hz}, 2^{\prime}-\mathrm{CH}_{3}\right), 2.44\left(2 \mathrm{H}, \mathrm{m},-\mathrm{CH}_{2}\right.$ in ethyl), $3.99\left(1 \mathrm{H}, \mathrm{m}, 2^{\prime}-\mathrm{CH}\right), 5.44$ $\left(1 \mathrm{H}, \mathrm{m}, 3^{\prime}-\mathrm{H}\right), 6.16(1 \mathrm{H}, \mathrm{s}, 3-\mathrm{H}), 6.97\left(1 \mathrm{H}, \mathrm{d}, J=2.4 \mathrm{~Hz}, 4^{\prime}-\mathrm{H}\right), 7.12(1 \mathrm{H}, \mathrm{d}, J=8.7 \mathrm{~Hz}, 6-\mathrm{H}), 8.05$ 
$(1 \mathrm{H}, \mathrm{d}, J=8.3 \mathrm{~Hz}, 5-\mathrm{H}) .[\alpha]_{\mathrm{D}}+135\left(c 0.1, \mathrm{CHCl}_{3}\right)$. HRMS(MALDI-DHB): Calcd. for $\mathrm{C}_{35} \mathrm{H}_{40} \mathrm{O}_{10} \mathrm{~S}$ : $675.2240\left[\mathrm{M}+\mathrm{Na}^{+}\right]$; Found: $675.2234\left[\mathrm{M}+\mathrm{Na}^{+}\right]$. 7a. mp $249-252^{\circ} \mathrm{C} ;{ }^{1} \mathrm{H} \mathrm{NMR}$ $\left(\mathrm{CDCl}_{3}, 400 \mathrm{MHz}\right) \delta 0.96-1.76\left(24 \mathrm{H}, 6 \times-\mathrm{CH}_{3}\right.$ in camphanoyl, $\left.2 \times 2^{\prime}-\mathrm{CH}_{3}\right), 1.25\left(3 \mathrm{H}, \mathrm{t},-\mathrm{CH}_{3}\right.$ in ethyl), 1.70-2.60 $\left(10 \mathrm{H}, \mathrm{m},-\mathrm{CH}_{2}\right.$ in ethyl, $4 \times-\mathrm{CH}_{2}$ in camphanoyl), $5.62\left(1 \mathrm{H}, \mathrm{d}, J=4.3 \mathrm{~Hz}, 3^{\prime}-\right.$ $\mathrm{CH}), 6.16(1 \mathrm{H}, \mathrm{s}, 3-\mathrm{H}), 6.96\left(1 \mathrm{H}, \mathrm{d}, J=4.3 \mathrm{~Hz}, 4^{\prime}-\mathrm{H}\right), 7.11(1 \mathrm{H}, \mathrm{d}, J=8.2 \mathrm{~Hz}, 6-\mathrm{H}), 8.06(1 \mathrm{H}, \mathrm{d}, J$ $=8.6 \mathrm{~Hz}, 5-\mathrm{H}) .[\alpha]_{\mathrm{D}}-130\left(c \mathrm{0.1}, \mathrm{CHCl}_{3}\right)$. HRMS(MALDI-DHB): Calcd. for $\mathrm{C}_{36} \mathrm{H}_{42} \mathrm{O}_{10} \mathrm{~S}$ : $689.2396\left[\mathrm{M}+\mathrm{Na}^{+}\right]$; Found: $689.2391\left[\mathrm{M}+\mathrm{Na}^{+}\right]$. 7b. mp $188-189^{\circ} \mathrm{C} ;{ }^{1} \mathrm{H} \mathrm{NMR}\left(\mathrm{CDCl}_{3}, 400 \mathrm{MHz}\right)$ $\delta 0.88-1.76\left(24 \mathrm{H}, 6 \times-\mathrm{CH}_{3}\right.$ in camphanoyl, $\left.2 \times 2^{\prime}-\mathrm{CH}_{3}\right), 1.26\left(3 \mathrm{H}, \mathrm{t},-\mathrm{CH}_{3}\right.$ in ethyl), 1.60-2.66 $\left(10 \mathrm{H}, \mathrm{m},-\mathrm{CH}_{2}\right.$ in ethyl, $4 \times-\mathrm{CH}_{2}$ in camphanoyl), $5.71\left(1 \mathrm{H}, \mathrm{d}, J=4.3 \mathrm{~Hz}, 3^{\prime}-\mathrm{CH}\right), 6.15(1 \mathrm{H}, \mathrm{s}, 3-$ H), $6.92\left(1 \mathrm{H}, \mathrm{d}, J=4.3 \mathrm{~Hz}, 4^{\prime}-\mathrm{H}\right), 7.10(1 \mathrm{H}, \mathrm{d}, J=8.6 \mathrm{~Hz}, 6-\mathrm{H}), 8.04(1 \mathrm{H}, \mathrm{d}, J=8.2 \mathrm{~Hz}, 5-\mathrm{H})$. $[\alpha]_{\mathrm{D}}+27\left(c\right.$ 0.1, $\left.\mathrm{CHCl}_{3}\right)$. HRMS(MALDI-DHB): Calcd. for $\mathrm{C}_{36} \mathrm{H}_{42} \mathrm{O}_{10} \mathrm{~S}: 689.2396\left[\mathrm{M}+\mathrm{Na}^{+}\right]$; Found: $689.2391\left[\mathrm{M}+\mathrm{Na}^{+}\right]$. 8a. $\mathrm{mp} 135-137^{\circ} \mathrm{C} ;{ }^{1} \mathrm{H} \mathrm{NMR}\left(\mathrm{CDCl}_{3}, 300 \mathrm{MHz}\right) \delta 1.11-1.13(18 \mathrm{H}$, $6 \times-\mathrm{CH}_{3}$ in camphanoyl), 1.56-2.53 $\left(8 \mathrm{H}, \mathrm{m}, 4 \times-\mathrm{CH}_{2}\right.$ in camphanoyl), $1.38\left(3 \mathrm{H}, \mathrm{s}, 2^{\prime}-\mathrm{CH}_{3}\right), 1.66$ $\left(3 \mathrm{H}, \mathrm{s}, 2^{\prime}-\mathrm{CH}_{3}\right), 2.40\left(3 \mathrm{H}, \mathrm{s}, 4-\mathrm{CH}_{3}\right), 5.63\left(1 \mathrm{H}, \mathrm{d}, J=4.5 \mathrm{~Hz}, 3^{\prime}-\mathrm{H}\right), 6.18(1 \mathrm{H}, \mathrm{d}, J=0.9 \mathrm{~Hz}, 3-\mathrm{H})$, $6.76\left(1 \mathrm{H}, \mathrm{d}, J=4.5 \mathrm{~Hz}, 4^{\prime}-\mathrm{H}\right), 7.04(1 \mathrm{H}, \mathrm{d}, J=8.7 \mathrm{~Hz}, 6-\mathrm{H}), 7.48(1 \mathrm{H}, \mathrm{d}, J=8.4 \mathrm{~Hz}, 5-\mathrm{H}) . \mathrm{HRMS}$ (MALDI-DHB) calcd mass for $\mathrm{C}_{35} \mathrm{H}_{40} \mathrm{O}_{10} \mathrm{~S}\left[\mathrm{M}^{+}-\mathrm{H}\right] 651.2269$, found 651.2270. 8b. mp 151$153^{\circ} \mathrm{C} ;{ }^{1} \mathrm{H}$ NMR $\left(\mathrm{CDCl}_{3}, 300 \mathrm{MHz}\right) \delta 0.87-1.15\left(18 \mathrm{H}, 6 \times-\mathrm{CH}_{3}\right.$ in camphanoyl), 1.64-2.64 (8H, $\mathrm{m}, 4 \times-\mathrm{CH}_{2}$ in camphanoyl), $1.38\left(3 \mathrm{H}, \mathrm{s}, 2^{\prime}-\mathrm{CH}_{3}\right), 1.73\left(3 \mathrm{H}, \mathrm{s}, 2^{\prime}-\mathrm{CH}_{3}\right), 2.40(3 \mathrm{H}, \mathrm{d}, J=1.5 \mathrm{~Hz}, 4-$ $\left.\mathrm{CH}_{3}\right), 5.69\left(1 \mathrm{H}, \mathrm{d}, J=4.5 \mathrm{~Hz}, 3^{\prime}-\mathrm{CH}\right), 6.17(1 \mathrm{H}, \mathrm{d}, J=1.5 \mathrm{~Hz}, 3-\mathrm{H}), 6.92\left(1 \mathrm{H}, \mathrm{d}, J=4.5 \mathrm{~Hz}, 4^{\prime}-\right.$ H), $7.04(1 \mathrm{H}, \mathrm{d}, J=8.1 \mathrm{~Hz}, 6-\mathrm{H}), 7.48(1 \mathrm{H}, \mathrm{d}, J=8.4 \mathrm{~Hz}, 5-\mathrm{H})$. HRMS (MALDI-DHB) calcd mass for $\mathrm{C}_{35} \mathrm{H}_{40} \mathrm{O}_{10} \mathrm{~S}\left[\mathrm{M}^{+}-\mathrm{H}\right] 651.2269$, found 651.2273 .

13. HIV-1 infectivity assay:Anti-HIV-1 activity was measured as reductions in Luc reporter gene expression after a single round of virus infection of TZM-bl cells. HIV-1 at 200 TCID $_{50}$ and various dilutions of test samples (eight dilutions, four-fold stepwise) were mixed in a total volume of $100 \mu \mathrm{L}$ growth medium in 96-well black solid plates (Corning-Costar). After 48-h incubation, culture medium was removed from each well and $100 \mu \mathrm{L}$ of Bright Glo luciferase reagent was added to each culture well. The luciferase activity in the assay wells was measured using a Victor 2 luminometer. The $50 \%$ inhibitory dose $\left(\mathrm{EC}_{50}\right)$ was defined as the sample concentration that caused a 50\% reduction in Relative Luminescence Units (RLU) compared to virus control wells after subtraction of background RLU. 


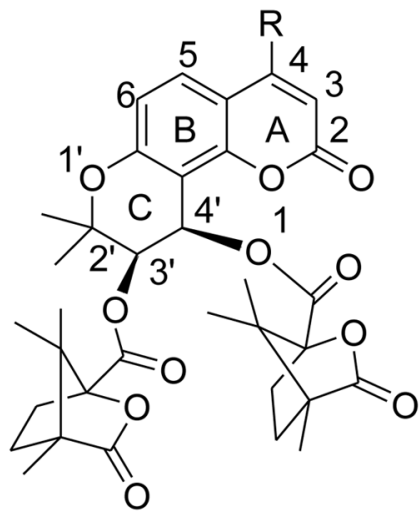

1. $D C K R=H$

3. 4-Methyl DCK $\mathrm{R}=\mathrm{CH}_{3}$

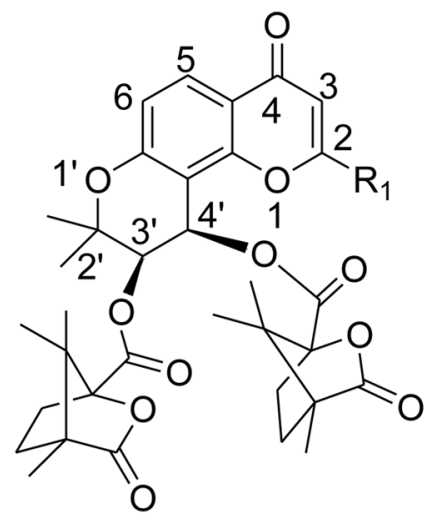

2. $D C P \quad R_{1}=H$

4. 2-Ethyl DCP $\mathrm{R}_{1}=\mathrm{CH}_{2} \mathrm{CH}_{3}$

Figure 1.

Structures of previously synthesized DCK and DCP analogues (1-4). 


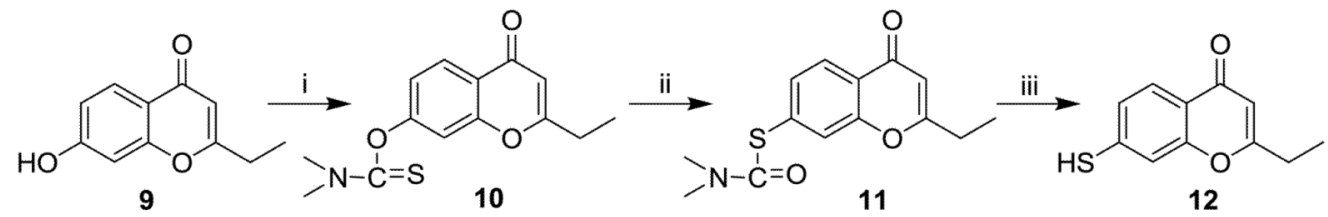

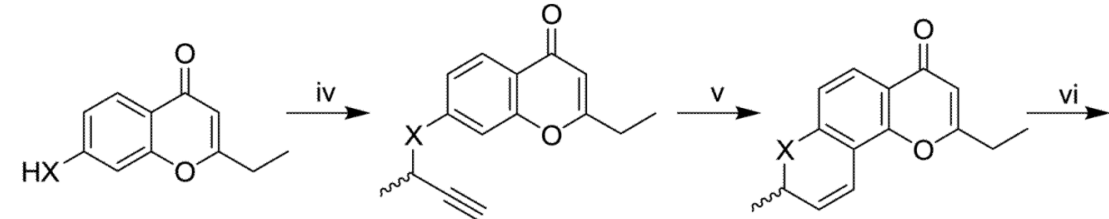

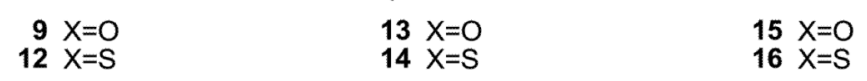

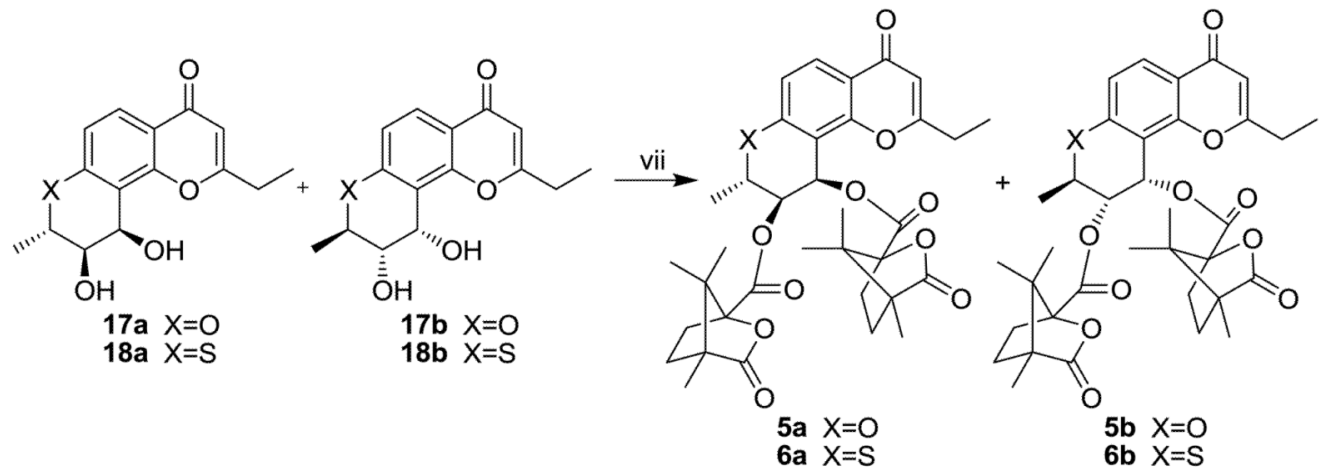

Scheme 1.

Reagents and conditions: (i) dimethylthiocarbamoyl chloride, EtOH, $\mathrm{K}_{2} \mathrm{CO}_{3}$, r.t.; (ii) 240 ${ }^{\circ} \mathrm{C}, \mathrm{N}_{2}$; (iii) $\mathrm{KOH}, \mathrm{CH}_{3} \mathrm{OH}, \mathrm{N}_{2}$, reflux; (iv) 3-chloro-1-butyne, $\mathrm{K}_{2} \mathrm{CO}_{3}$, $\mathrm{KI}$ in DMF or acetone, r.t.; (v) $N, N$-diethylaniline, reflux; (vi) $\mathrm{K}_{2} \mathrm{OsO}_{2}(\mathrm{OH})_{4},(\mathrm{DHQ})_{2}-\mathrm{PHAL}, \mathrm{K}_{3} \mathrm{Fe}(\mathrm{CN})_{6}$, $\mathrm{K}_{2} \mathrm{CO}_{3}$ in $t$-butanol $/ \mathrm{H}_{2} \mathrm{O}(\mathrm{v} / \mathrm{v}=1: 1)$, ice bath; (vii) $(S)$-camphanic chloride, DMAP in $\mathrm{CH}_{2} \mathrm{Cl}_{2}$, r.t. 


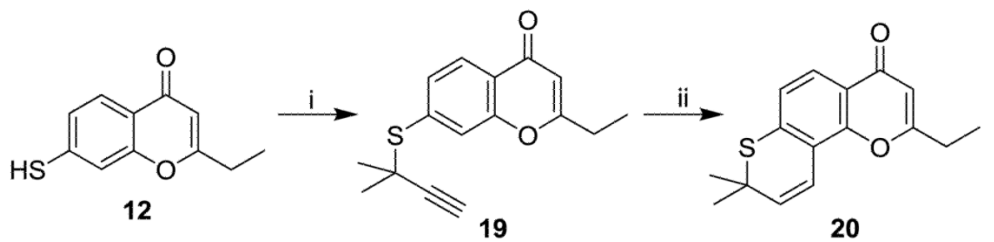

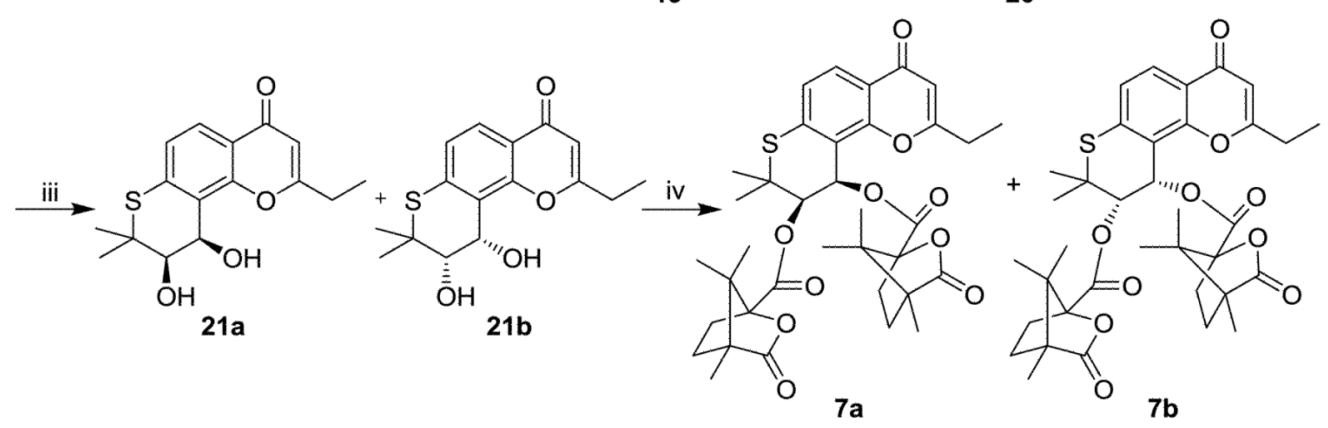

Scheme 2.

Reagents and conditions: (i) 3-chloro-3-methyl-1-butyne, $\mathrm{KOH}, \mathrm{N}_{2}, \mathrm{EtOH} / \mathrm{H}_{2} \mathrm{O}$ (v/v=1:1), r.t.; (ii) $N, N$-diethylaniline, reflux; (iii) $\mathrm{K}_{2} \mathrm{OsO}_{2}(\mathrm{OH})_{4}$, (DHQ) $)_{2}-\mathrm{PHAL}, \mathrm{K}_{3} \mathrm{Fe}(\mathrm{CN})_{6}, \mathrm{~K}_{2} \mathrm{CO}_{3}$ in $t$-butanol/ $\mathrm{H}_{2} \mathrm{O}(\mathrm{v} / \mathrm{v}=1: 1)$, ice bath; (iv) (S)-camphanic chloride, DMAP in $\mathrm{CH}_{2} \mathrm{Cl}_{2}$, r.t. 
<smiles>C#CC(C)(C)Sc1ccc2c(C)cc(=O)oc2c1</smiles>

22

23

24<smiles>Cc1cc(=O)oc2c3c(ccc12)SC(C)(C)[C@H](O)[C@H]3O</smiles>

Scheme 3.

Reagents and conditions: (i) 3-chloro-3-methyl-1-butyne, $\mathrm{KOH}$ in EtOH, $\mathrm{N}_{2}$; (ii) $\mathrm{N}, \mathrm{N}$ -

diethylaniline, reflux; (iii) $\mathrm{K}_{2} \mathrm{OsO}_{2}(\mathrm{OH})_{4}$, (DHQ) 2 - $\mathrm{PHAL}, \mathrm{K}_{3} \mathrm{Fe}(\mathrm{CN})_{6}, \mathrm{~K}_{2} \mathrm{CO}_{3}$ in $t$-butanol/ $\mathrm{H}_{2} \mathrm{O}\left(\mathrm{v} / \mathrm{v}=1: 1\right.$ ), ice bath; (iv) (S)-camphanic chloride, DMAP in $\mathrm{CH}_{2} \mathrm{Cl}_{2}$. 
Table 1

Anti-HIV-1 NL4-3 data of analogues 5-8 in TZM-bl cells ${ }^{a}$

\begin{tabular}{cccc}
\hline Compound & $\mathbf{C C}_{\mathbf{5 0}}(\boldsymbol{\mu M})$ & $\mathbf{E C}_{\mathbf{5 0}}(\boldsymbol{\mu M})$ & $\mathbf{T I}$ \\
\hline $\mathbf{5 a}$ & 4.55 & 0.030 & 153 \\
$\mathbf{5 b}$ & - & - & $\mathrm{NS}$ \\
$\mathbf{6 a}$ & 1.84 & 0.038 & 48.0 \\
$\mathbf{6 b}$ & 3.83 & 0.184 & 20.8 \\
$\mathbf{7 a}$ & 5.4 & 0.054 & 100 \\
$\mathbf{7 b}$ & - & - & $\mathrm{NS}$ \\
$\mathbf{8 a}$ & $>30.6$ & 0.128 & $>238$ \\
$\mathbf{8 b}$ & $>30.6$ & 8.59 & $>3.6$ \\
$\mathbf{2 - E t h y l - D C P}$ & 14.3 & 0.12 & 119 \\
\hline
\end{tabular}

${ }^{a}$ All data presented in this table were averaged from at least three independent experiments. EC50: concentration that inhibits NL4-3 replication by $50 \%$. CC50: concentration that inhibits uninfected TZM-bl cell growth by $50 \%$. $\mathrm{TI}=\mathrm{CC}_{50} / \mathrm{EC}_{50}$. NS: there was no inhibition at concentrations below the $\mathrm{CC}_{50}$. 